\title{
Genome-wide analysis of glyoxalase-like gene families in grape (Vitis vinifera L.) and their expression profiling in response to downy mildew infection
}

Tiemei Li $i^{1,2,3}$, Xin Cheng ${ }^{1,2,3}$, Yuting Wang ${ }^{1,2,3}$, Xiao Yin ${ }^{1,2,3}$, Zhiqian Li ${ }^{1,2,3}$, Ruiqi Liu ${ }^{1,2,3}$, Guotian Liu ${ }^{1,2,3}$, Yuejin Wang ${ }^{1,2,3}$ and Yan $\mathrm{Xu}^{1,2,3^{*}}$

\begin{abstract}
Background: The glyoxalase system usually comprises two enzymes, glyoxalase I (GLYI) and glyoxalase II (GLYII). This system converts cytotoxic methylglyoxal (MG) into non-toxic D-lactate in the presence of reduced glutathione (GSH) in two enzymatic steps. Recently, a novel type of glyoxalase III (GLYIII) activity has observed in Escherichia coli that can detoxify MG into D-lactate directly, in one step, without a cofactor. Investigation of the glyoxalase enzymes of a number of plant species shows the importance of their roles in response both to abiotic and to biotic stresses. Until now, glyoxalase gene families have been identified in the genomes of four plants, Arabidopsis, Oryza sativa, Glycine max and Medicago truncatula but no similar study has been done with the grapevine Vitis vinifera L.

Results: In this study, four GLYI-like, two GLYII-like and three GLYIII-like genes are identified from the genome database of grape. All these genes were analysed in detail, including their chromosomal locations, phylogenetic relationships, exon-intron distributions, protein domain organisations and the presence of conserved binding sites. Using quantitative real-time PCR analysis (qRT-PCR), the expression profiles of these genes were analysed in different tissues of grape, and also when under infection stress from downy mildew (Plasmopara viticola). The study reveals that most $V_{V} G L Y$-like genes had higher expressions in stem, leaf, tendril and ovule but lower expressions in the flower. In addition, most of the $V V G L Y$-like gene members were $P$. viticola responsive with high expressions 6-12 h and $96-120 \mathrm{~h}$ after inoculation. However, $V_{V G L Y l-l i k e 1}$ was highly expressed $48 \mathrm{~h}$ after inoculation, similar to VVPR1 and VVNPR1 which are involved in the defence response.

Conclusions: This study identified the GLYI-like, GLYII-like and GLYIII-like full gene families of the grapevine. Based on a phylogenetic analysis and the presence of conserved binding sites, we speculate that these glyoxalase-like genes in grape encode active glyoxalases. Moreover, our study provides a basis for discussing the roles of $V V G L Y I-$ like, VVGLYII-like and VVGLYII-like genes in grape's response to downy mildew infection. Our results shed light on the selection of candidate genes for downy mildew tolerance in grape and lay the foundation for further functional investigations of these glyoxalase genes.
\end{abstract}

Keywords: Glyoxalase, Methylglyoxal, Grapevine, Downy mildew, qRT-PCR

\footnotetext{
* Correspondence: yan.xu@nwsuaf.edu.cn

${ }^{1}$ College of Horticulture, Northwest A\&F University, Yangling, Shaanxi,

People's Republic of China

${ }^{2}$ State Key Laboratory of Crop Stress Biology for Arid Areas, Northwest A\&F

University, Yangling, Shaanxi, People's Republic of China

Full list of author information is available at the end of the article
}

(c) The Author(s). 2019 Open Access This article is distributed under the terms of the Creative Commons Attribution 4.0 International License (http://creativecommons.org/licenses/by/4.0/), which permits unrestricted use, distribution, and reproduction in any medium, provided you give appropriate credit to the original author(s) and the source, provide a link to the Creative Commons license, and indicate if changes were made. The Creative Commons Public Domain Dedication waiver (http://creativecommons.org/publicdomain/zero/1.0/) applies to the data made available in this article, unless otherwise stated. 


\section{Background}

The glyoxalase system is an almost ubiquitous pathway for the detoxification of methylglyoxal (MG) in living systems. The typical glyoxalase system can detoxify the highly cytotoxic compound, MG to the nontoxic D-lactate through the sequential activity of two enzymes - glyoxalase I (GLYI; lactoylglutathione lyase) and glyoxalase II (GLYII; hydroxyacylglutathione hydrolase). First, MG is converted into hemithioacetal (HTA) in the presence of reduced glutathione (GSH), then GLYI catalyses the HTA isomerisation into S-D-lactoyl-glutathione (SLG). Subsequently, SLG is hydrolysed to D-lactate by GLYII, recycling GSH to the system [1, 2]. Recently, a one-step detoxification of MG has been proposed for a few organisms. This is mediated by glyoxalase III (GLYIII) enzyme without any cofactor [3-7].

Glyoxalase family identification has so far been carried out in four plant genomes Arabidopsis, Oryza sativa, Glycine max and Medicago truncatula [8-10]. These glyoxalase gene identifications have been based on sequence analysis. In the genome wide analysis, eleven GLYI genes have been described in Arabidopsis thaliana and in rice [8]. However, further study revealed that only three Arabidopsis thaliana genes (AtGLYI2, AtGLYI3 and AtGLYI6) and two rice genes (OsGLYI8 and OsGLYI11.2) were functional and contained all the binding sites required for glyoxalase activity. In addition, the activity of AtGLYI2 was dependent on $\mathrm{Zn}^{2+}$, while the activities of AtGLYI3, AtGLYI6 and OsGLYI11.2 were dependent on $\mathrm{Ni}^{2+}$ [11-13]. Specifically, OsGLYI-8 did not show any strict requirement for a metal ion for its activity [14]. Similarly, two out of five GLYII proteins from Arabidopsis thaliana (AtGLYII2 and AtGLYII5) and two out of three GLYII proteins from rice (OsGLYII2 and OsGLYII3) showed GLYII enzyme activity [15-20]. Glyoxalase II of all species, including human, yeast and Arabidopsis, contains a highly conserved metal binding motif $(\mathrm{TH} X \mathrm{H} X \mathrm{DH})$ that is also present in the family of metallo- $\beta$-lactamases, which are known to require $\mathrm{Zn}$ (II) [21-23]. However, the glyoxalase III enzyme does not require any cofactor and is reported to be a member of the DJ-1/Pfp1 (PARK7/Pyrococcus furiosus protease I) superfamily with a conserved catalytic triad Glu-Cys-His in its active site. Mutagenesis studies of Hsp31 revealed that the Cys-185 and Glu-77 were essential for catalysis, whereas His-186 was less crucial for enzymatic function, although it participates in the catalytic process $[4,24]$. Site-directed mutagenesis of the conserved cysteine in the $\mathrm{N}$-terminal domain of OsDJ-1C, which can utilise MG as substrate to produce D-lactate in a glutathione-independent manner in rice, resulted in the loss of GLYIII activity. This confirms it is a functional enzyme and that cysteine is necessary for its activity [7].
The glyoxalase system has been well studied in the animal kingdom and shown to play numerous roles in cell division and proliferation, microtubule assembly, embryogenesis, maturation and cell death [2]. An active plant glyoxalase enzyme was first reported in Douglas fir needles [25]. Thereafter, several studies have reported the presence of glyoxalase activity in both monocotyledons and dicotyledons [26-31]. It was found that over-expressed GLYI and/or GLYII in plants significantly increased their tolerance to various abiotic stresses such as salinity, drought, extreme temperature and heavy-metal toxicity [32-36]. Thus, glyoxalases have been suggested as biomarkers for plant stress resistance [31, 37]. In addition to abiotic stress responses, glyoxalase genes have also been reported to be regulated by biotic stresses. A rice GLYI gene was found to be down-regulated after infection by Xanthomonas oryzae pv. oryzae or by Pyricularia grisea [38]. Proteomic comparison analysis of maize kernel embryos showed significant up-regulation of GLYI protein in the resistant embryos in response to Aspergillus flavus infection [39]. Similarly, induction of GLYI activity was observed in rice in response to attack by brown planthopper (Nilaparvata lugens) and in Brassica after infection by Sclerotinia sclerotiorum. After fungal infection, intercellular MG levels were significantly increased in susceptible genotypes, so high GLYI activity was crucial for the detoxification of MG $[39,40]$.

The grapevine (Vitis vinifera L.) is a worldwide cultivated crop of high economic value. Sequencing of the highly homozygous grapevine PN40024 genome provided the opportunity to analyse the grapevine genome and to identify gene families [41]. Grape downy mildew, caused by the oomycete Plasmopara viticola, occurs in most parts of the world, especially in those where it is wet during the vegetative growth period. A major outbreak of the disease can cause severe losses in both yield and berry quality [42]. Genetic and gene expression profiling analyses show that Rpv1, NPR1 homologs, and PR protein encoding genes contribute to the function of downy mildew resistance in grapevine [43-45]. Moreover, overexpression of VpPR10.1 can enhance the transgenic grape plants resistance to downy mildew $[46,47]$. It is important to find more crucial genes for grape downy mildew tolerance. In this study, we identify the gene families of the glyoxalase system in $V$. vinifera and discuss their roles in response to downy mildew. The existence of glyoxalases as multigene families in grape suggests there could be a number of undiscovered roles for these genes.

\section{Results}

Identification and detailed analysis of glyoxalase families in grape

To identify all the glyoxalase proteins in grape, lactoylglutathione lyase domain (PF00903), metallo-beta-lactamase 
domain (PF00753) and DJ-1/PfpI (PF01965) were downloaded from Pfam and used as queries to search the Grape Genome Database using HMMER with default E-values (< 1.0) [48]. The proteins were then manually confirmed using Pfam and BLASTP along with the previously reported GLYI proteins of Arabidopsis, rice, soybean and M. truncatula [8-10]. Proteins were identified that contained the glyoxalase domain (PF00903) and that contained all four conserved binding sites required for glyoxalase activity- i.e. (a) the active site, (b) the metal binding site, (c) the GSH binding site and (d) the dimer interface. These proteins were classified as VvGLYI-like (Table 1 and Additional file 1: Table S1) and those without all the four conserved binding sites were identified as non-glyoxalases (Additional file 2: Table S2) $[8,11]$. Proteins that contained the metallo-betalactamase domain (PF00753) along with conserved metal binding sites (THXHXDH/H/D/H), an active site (C/GHT) and seven conserved GSH binding sites $(\mathrm{C} / \mathrm{K}(\mathrm{R}) / \mathrm{F}(\mathrm{Y}) / \mathrm{Y} / \mathrm{N} /$ $\mathrm{R} / \mathrm{K}$ ) and that had a putative hydroxyacyl glutathione hydrolase function were classified as VvGLYII-like (Table 1, Additional file 3: Table S3 and Additional file 4: Table S4) [8-10, 49-51]. Likewise, proteins that contained the DJ-1/PfpI (PARK7/Pyrococcus furiosus protease I) domain (PF01965) and Glu-Cys-His/Tyr catalytic triad were classified as VvGLYIII-like [6, 10]. A total of four GLYI-like, two GLYII-like and three GLYIII-like proteins were identified in the grape genome. These were named VvGLYI-like1 to VvGLYI-like4, VvGLYII-like1 to VvGLYII-like2 and VvGLYIII-like1 to VvGLYIII-like3 according to their location order on the grape chromosomes [8]. Meanwhile, all the grape glyoxalase-like genes identified were analysed in detail (Table 1). They were found to be located on eight different chromosomes. The CDS length of the $V v G L Y$-like members varied from $708 \mathrm{bp}$ to 1344 bp. The largest protein was VvGLYIII-like1 with a length of $447 \mathrm{aa}$, while the smallest was VvGLYI-like2 with a length of 235 aa (Table 1). Detailed information on the glyoxalase genes in grape are listed in Table 1, including their accession numbers, protein lengths, locations and similarities to the Arabidopsis's orthologues.

\section{Protein motif identification and gene structure analysis of glyoxalase-like families of grape}

The exon-intron structure of the VvGLYI-like, VvGLYIIlike and VvGLYIII-like genes was varied (Fig. 1). There were eight exons in VvGLYI-like3 and VvGLYI-like2 genes (Fig. 1), while VvGLYI-like1 and VvGLYI-like4 both had nine exons. The numbers of exons in VvGLYII-like1 and VvGLYII-like2 were eight and seven respectively (Fig. 1). VvGLYIII-like1 and VvGLYIII-like3 each had eight exons, while VvGLYIII-like2 had five exons (Fig. 1). Genes with longer coding sequences are significantly more important and evolve more slowly than genes with shorter CDSs and they contain more functional domains within the gene [52].

All predicted VvGLYI-like proteins were analysed using Pfam to identify the glyoxalase domain (PF00903). Results indicate that VvGLYI-like1 and VvGLYI-like4 contain two glyoxalase domains while the other proteins had only a single PF00903 domain (Fig. 1). Analysis of the VvGLYIIlike proteins showed that they both had a metallo-beta-lactamase domain along with a hydroxyacylglutathione hydrolase C-terminus domain (HAGH-C) (PF16123) (Fig. 1). The HAGH-C domain was a substrate binding site usually found in the C-terminus of GLYII proteins along with the catalytic domain (PF00753) [53]. The previously reported DJ-1 proteins of rice and Arabidopsis all contain two DJ-1/PfpI domains, while the DJ-1 proteins of E. coli, Drosophila, Caenorhabditis elegans and human contain only one $[5,7]$. Structural analysis of the three grape GLYIII-like proteins show they all have two DJ-1/ PfpI domains (Fig. 1). Unlike human and other homologs, the plant glyoxalases seem to have higher efficiency or better regulatory properties. Thus, plants seem to have greater adaptability to their ever-changing environments from which they are unable to escape.

\section{Conserved binding sites and phylogenetic analysis of operative GLYI proteins}

GLYI is a metalloenzyme with conserved metal binding sites of $\mathrm{H} / \mathrm{QEH} / \mathrm{QE}$, requiring divalent metal ions $\left(\mathrm{Ni}^{2+} / \mathrm{Co}^{2+}\right.$ or $\left.\mathrm{Zn}^{2+}\right)$ for activation. It has been reported that GLYIs dependent on $\mathrm{Zn}^{2+}$ have longer domains (more than $140 \mathrm{aa}$ ) than $\mathrm{Ni}^{2+} / \mathrm{Co}^{2+}$ dependent ones (around 120 aa) and have unique regions in their sequences [11, 12, 54]. To determine metal ion dependency, the N-terminal lactoylglutathione lyasedomains of the operative GLYI proteins were aligned using ClustalW along with the human GlyI domain [55] (Additional file 5: Figure S1). Among the four VvGLYI proteins, VvGLYIlike1, VvGLYI-like3 and VvGLYI-like4 were predicted to be $\mathrm{Ni}^{2+}$-dependent because their domains were around 120 aa and specific $\mathrm{Zn}^{2+}$-dependent regions were absent. Meanwhile, VvGLYI-like2 was predicted to be $\mathrm{Zn}^{2+}$-dependent because of its domain length of 151 aa and because it had conserved regions for $\mathrm{Zn}^{2+}$-dependence (Additional file 1: Table S1 and Additional file 5: Figure $\mathrm{S} 1)$.

To gain a broader understanding of the phylogeny of GLYIs, an unrooted phylogenetic tree was constructed using the Maximum Likelihood (ML) method, and based on the multiple sequence alignment of the lactoylglutathione lyase domains of the proteins listed in Additional file 1: Table S1 (Fig. 2a). According to the phylogenetic tree, the putative GLYI proteins can be divided into two subfamilies (I and II) (Fig. 2a). Subfamily I contains all the putative $\mathrm{Ni}^{2+}$-dependent GLYI proteins while the putative 


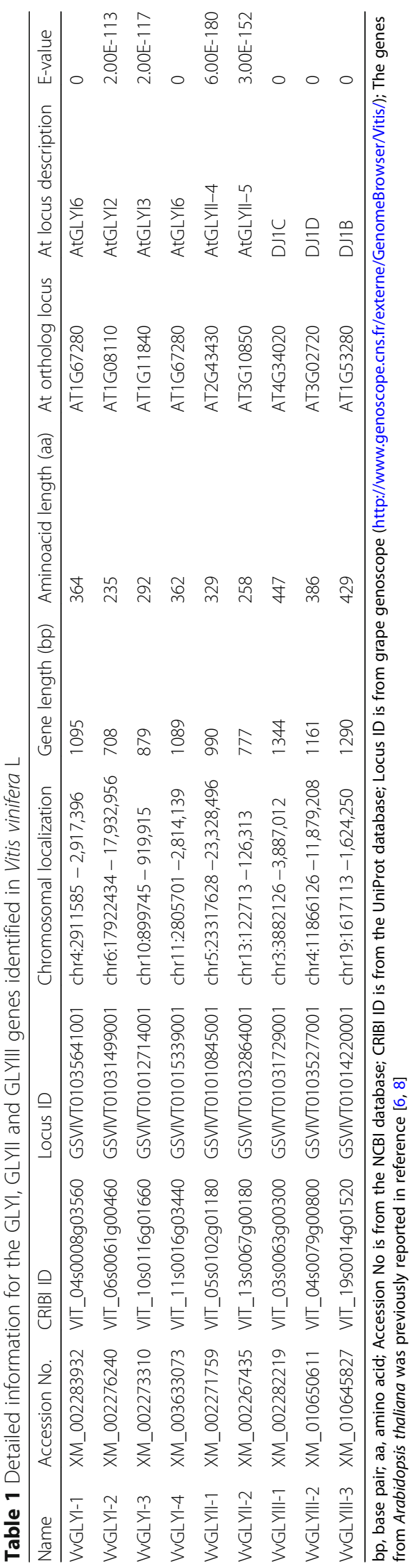





Fig. 1 Gene structures of grape glyoxalase genes and domain architectures of grape glyoxalase proteins. The exons are represented by black boxes while the introns are represented by black lines. Only the exons are drawn to scale because some introns are too long. The glyoxalase domain (PF00903) of VVGLYI-like is represented by an orange box. For VVGLYII-like proteins, $\beta$-lactamase domain is represented by a blue box and the hydroxyacylglutathione hydrolase C-terminus (HAGH-C) domain is represented by an orange hexagon. The DJ/Pfpl domain (PF01965) of VVGLYIII-like protein is represented by a dark green box. The domain position is indicated by the number inside the box or hexagon. The full length of protein is indicated by the numbers at both ends

$\mathrm{Zn}^{2+}$-dependent GLYI proteins were in subfamily II. This provides a basis for our future research on gene functions.

\section{Conserved sites and phylogenetic analysis of putative GLYII proteins}

The full length of the VvGLYII-like proteins and of the previously reported GLYII proteins of Arabidopsis, rice, soybean and $M$. truncatula [8-10] were manually analyzed using BLASTP and multiple sequence alignment to analyse for the presence of conserved binding sites required for GLYII activity (Additional file 3: Table S3, Additional file 4: Table S4 and Additional file 6: Figure S2). The putative GLYII-like proteins in which all binding sites are present are listed in Additional file 3: Table S3. We speculate that these proteins should be studied further with regard to their roles as active GLYII proteins.

We constructed another tree using the ML method, based on multiple sequence alignment of the full length of the putative GLYII proteins listed in Additional file 3: Table S3 from grape, Arabidopsis, rice, soybean and $M$. truncatula [8-10] (Fig. 2b). This tree was divided into two subgroups. VvGLYII-like1 is in the branch I, along with AtGLYII-5 which has been reported to be an active GLYII protein located in the mitochondria and to have a predominant metal center of Fe(III)Zn(II) but does not seem specifically to bind manganese [18]. VvGLYII-like2 is in branch II, along with AtGLYII-2 which is reported to be an active GLYII protein located in the cytosol and that has been shown to bind a mixture of $\mathrm{Zn}$, Fe or Mn [21]. We speculate that these proteins in the two branches may be located in different places in the cell and have different metal dependences (Fig. 2b).
Phylogenetic analysis of putative GLYIII proteins It has been reported that both DJ-1and Hsp31 proteins contain the Glu-Cys-His catalytic triad. Among these, the glutamate and cysteine residues in the same locations in the two enzymes directly involved in catalysis are essential for GLYIII enzymatic activity. In contrast, histidine residues at different sequence and structural positions, are less important to enzyme activity [4, 5]. Previously reported DJ-1 protein sequences from Arabidopsis, rice, soybean and $M$. truncatula along with VvGLYIII-like proteins were aligned using ClustalW [9, 10, 56]. Incomplete sequences and proteins not containing the highly conserved glutamate and cysteine residues either in the $\mathrm{N}$-terminal or C-terminal were discarded (Additional file 7: Figure S3). The remaining sequences were used for phylogenetic analysis. Using the MEGA 5.1 tool, a ML tree of the N-terminal and C-terminal DJ-1/PfpI domains from grape, Arabidopsis, rice soybean and $M$. truncatula along with the well characterised DJ-1/Hsp31 proteins from human, mouse, Drosophila, C. elegans and E. coli (Fig. 2c). This tree showed three major clades, I to III. Clade II contained all the DJ-1 proteins from the animal species. In Clade III, heat-inducible molecular chaperones (Hsp31) from E. coli, AtDJ-1D and OsDJ-1C have been shown in previous studies to be a primary GLYIII enzyme $[4,6,7]$. Thus VvGLYIII-like2 in this clade is speculated to be an important GLYIII enzyme worthy for further study. In Clade I, AtDJ-1a and AtDJ-1b exhibited weak specificity towards MG but higher specificity towards glyoxal and AtDJ-1a was involved in the oxidative stress response $[23,57]$. Thus, we speculate that VvGLYIII-lik1 and VvGLYIII-lik3 in Clade I may exhibit weak glyoxalase activities or have different functions from glyoxalase. 


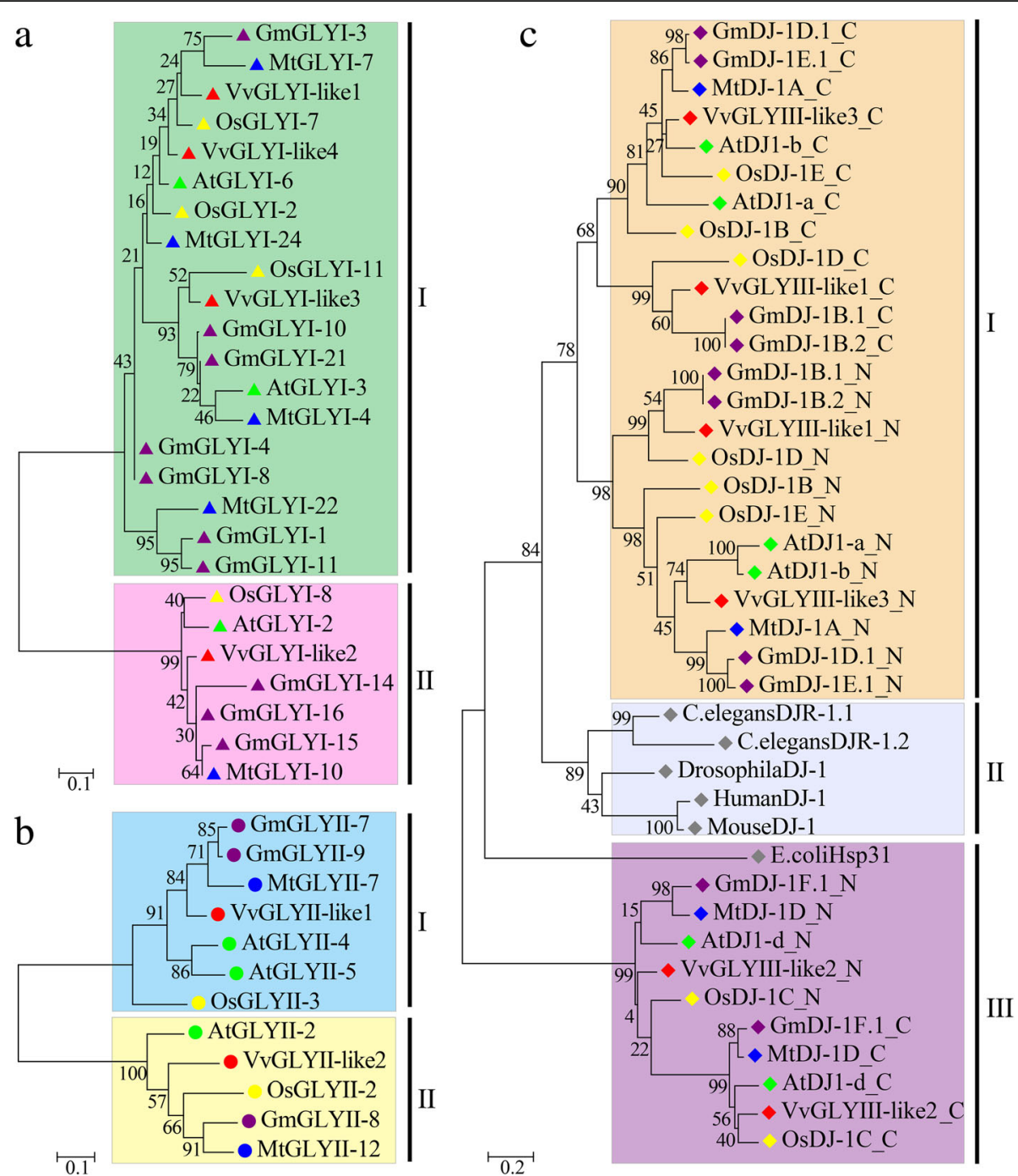

Fig. 2 Phylogenetic relationships of GLY-like proteins from various plant species. Unrooted trees were produced using MEGA5.1 software with the Maximum Likelihood method and 1000 bootstrap replicates using the N-term domain of GLYls (a), full length amino acid sequences of GLYlls (b) and the two domains of GLYIIIs respectively. Glyoxalase protein sequences are provided as Additional files 13, 14 and 15. Members of the glyoxalase gene families from grape, Arabidopsis, rice, soybean and Medicago truncatula are marked by red, green, yellow, purple and blue respectively. The domains of human, mouse, Drosophila, Caenorhabditis elegans and Escherichia coli are marked with a white rhombus. The bootstrap values are shown near the nodes. Scale bar represents 0.1 or 0.2 amino acid substitution per site

Expression profiles of grape glyoxalase genes in different tissues and under downy mildew stress

To investigate the expression of glyoxalase genes in the different tissues of grape, we analysed the expressions of glyoxalase genes in shoots, stems, leaves, flowers, tendrils and ovules at 20/30 and 40 days after flowering (DAF) in $V$. vinifera $\mathrm{L}$. cv. 'Thompson seedless' by qRT-PCR. $V v M A D S 9$ which is specifically expressed in flowers, served as the positive control $[58,59]$. Based on the expression profiles, all the glyoxalase genes were expressed in ovules and most had higher expressions in stems, leaves, tendrils, but lower expressions in flowers. However, some differences were noted. For instance,
$V \nu G L Y I-4$ had lower expressions in roots, stems, leaves than in tendrils and ovules (Fig. 3). With confirmation of the expressions of glyoxalase-like genes in grape, PR1 and NPR1 (involved in the defence response) and $V \nu G A P H D$ (a housekeeping gene) were evaluated by qRT-PCR following infection with P. viticola (Fig. 4). There were no significant differences in the expressions of $V v G A P H D$ after infection. This indicates our methods are reliable. The expressions of $P R 1$ and NPR1 were found to increase 24-48 h after inoculation. However, VvGLYI-like2, VvGLYI-like3, VvGLYI-like4, VvGLYII-like2 and all three $V v G L Y Y I I I-l i k e$ genes were first expressed at 6-12 h, expression then decreased but increased again 


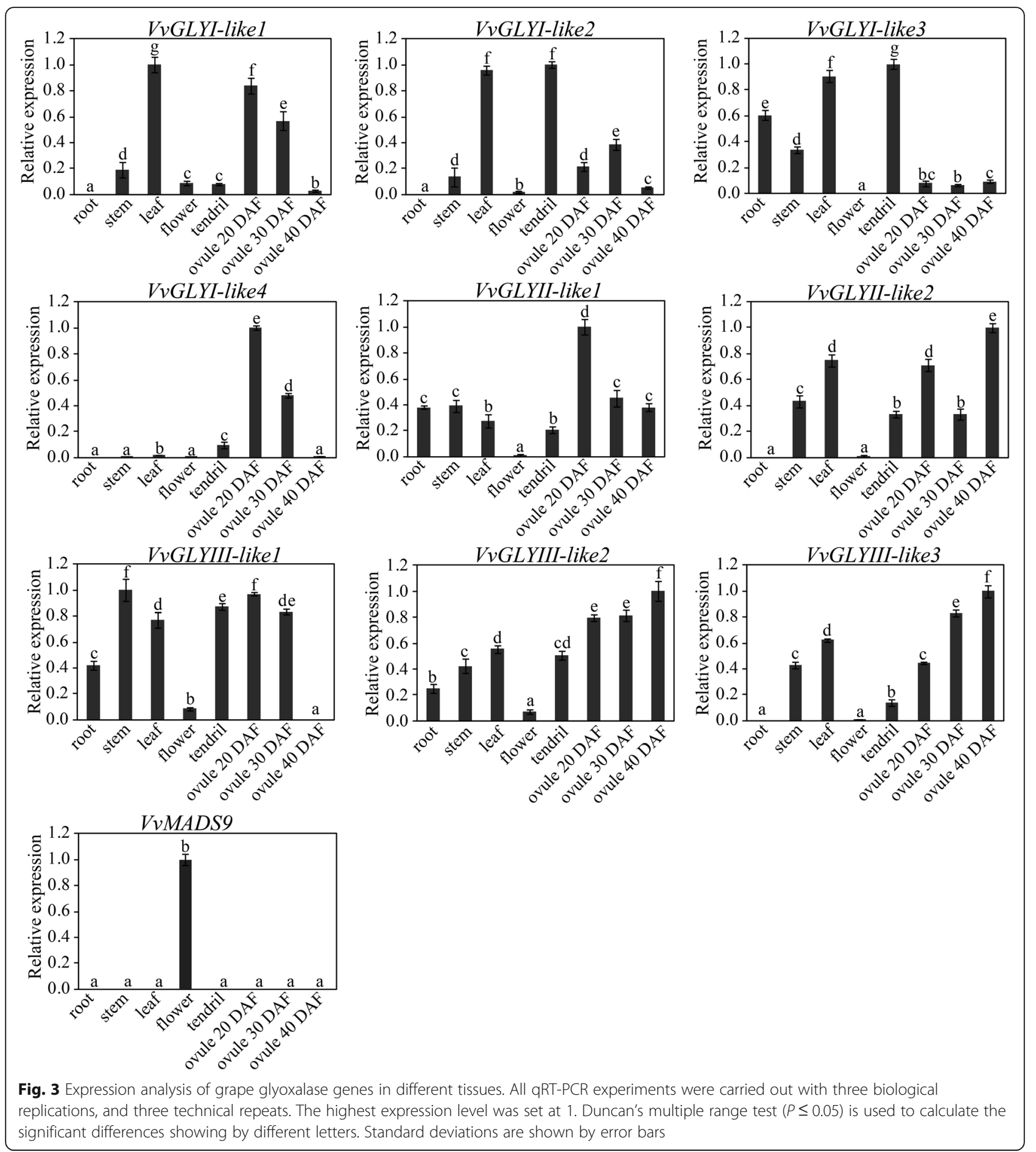

at $96-120 \mathrm{~h}$, while VvGLYII-like1 was down-regulated. Meanwhile, VvGLYI-like1 was expressed most strongly at $48 \mathrm{~h}$ but like PR1 and NPR1, expression then decreased. It is speculated that $V v G L Y I-l i k e 1$ could play an important role in grape's defence response to P. viticola (Fig. 4). The expression patterns of the various glyoxalase members are different. This indicates the various glyoxalase members play different roles in grape downy mildew stress modulation pathway.

\section{Discussion}

Identification of glyoxalase genes in plants

Genome-wide analysis of the glyoxalase enzymes have been carried out previously in rice, Arabidopsis, soybean 


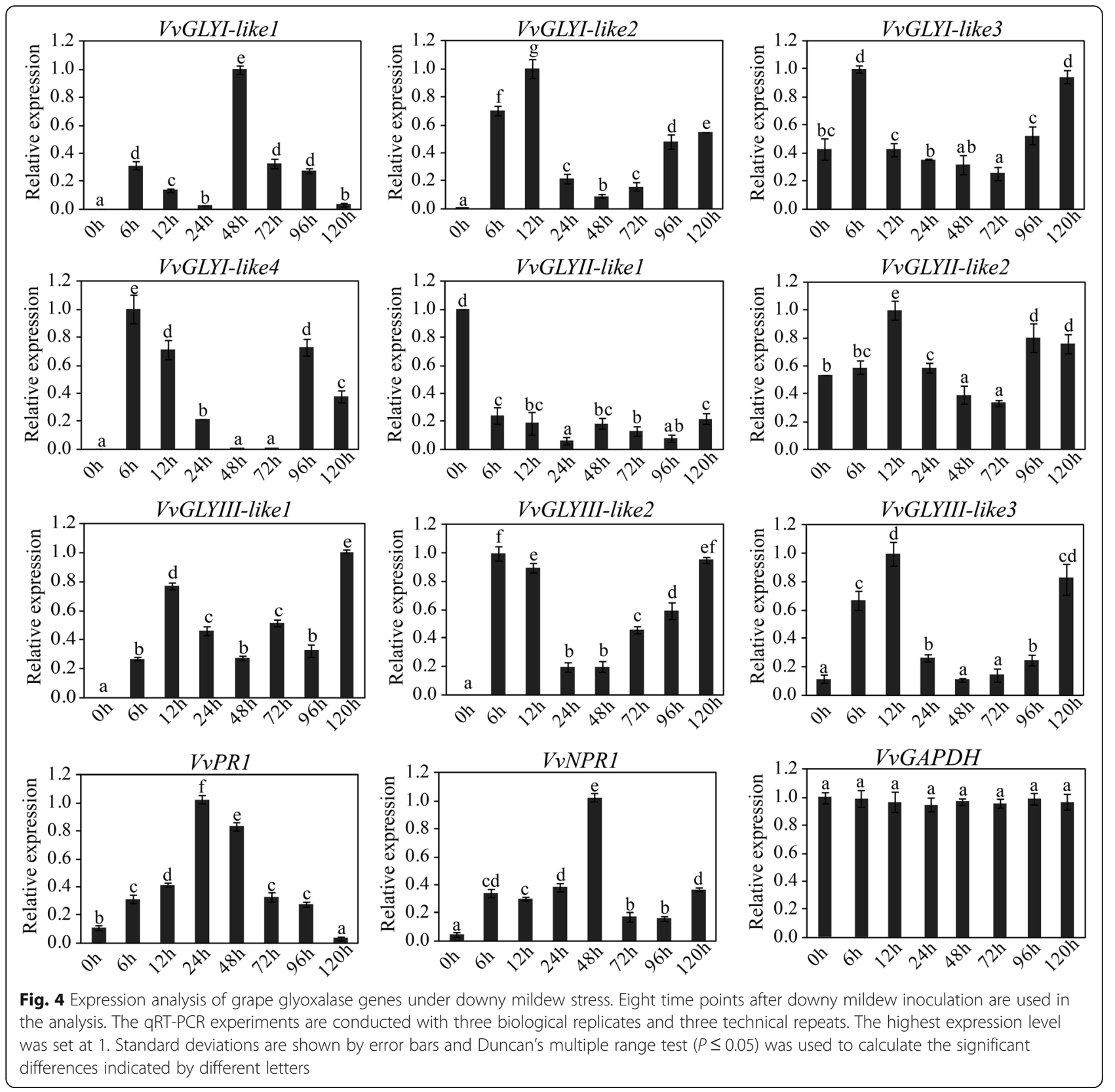

and M. truncatula [8-10]. However, these families have not been studied in grape. Previous studies on GLYIs and GlYIIs in soybean and M. truncatula based on the presence of conserved metal ion binding sites and substrate binding sites to determine whether a protein sequence had enzyme activity $[9,10]$. However, the screening criterias used in these studies were not comprehensive. Jain et al. screened GLYI-like gene sequences in Arabidopsis and determined they contained not only metal binding sites, but also GSH binding sites, active sites and dimer interfaces [11]. Limphong et al. characterised GlYIIs in Arabidopsis and identified 14 amino acids that are important for substrate binding, metal binding and catalytic activity [49].
Using the new screening criteria, the present study identifies four GLYI-like proteins and two GLYII-like proteins in grape, 10 GLYI-like proteins and three GLYII-like proteins in soybean and five GLYI-like proteins and two GLYII-like proteins in $M$. truncatula. The number of previously reported proteins in full families and putative active members of all five plant species are listed in Additional file 8: Table S5, including the length of their genomes [60-64]. Reports on the structural properties of plant GLYIII proteins are few, with structure reported only for Arabidopsis AtDJ-1d protein, the conserved binding site was Glu-Cys-His triad [6]. Similarly, OsDJ-1C, a rice DJ-1 protein, generated through homology modelling 
studies using AtDJ-1d as a template, also had the conserved three-dimensional structure [7]. These glyoxalase families display wide differences in their sequences which might lead to diversity in their biochemical functions. However, at this stage, little is known about plant DJ-1 proteins.

\section{Predicted metal ion dependence properties of glyoxalases}

There are two types of metal dependency of GLYI proteins, $\mathrm{Zn}^{2+}$ and non- $\mathrm{Zn}^{2+}$ (mainly $\mathrm{Ni}^{2+}$ ). Regardless of the metal activation, both types have four conserved metal-binding residues (H/QEH/QE) $[13,57,65]$. Interestingly, metal dependence specificity of predicted GLYI proteins can easily be identified based on the length and sequence of proteins. The amino acid length of $\mathrm{Zn}^{2+}$-activated GLYI proteins is greater than of $\mathrm{Ni}^{2+}$-activated GLYI proteins and there is also a unique region in the sequence of $\mathrm{Zn}^{2+}$-activated proteins [11, 13, 56]. According to these criteria, in this study, three out of the four VvGLYI-like proteins are likely to be $\mathrm{Ni}^{2+}$-dependent and VvGLYI-like2 was speculated to be $\mathrm{Zn}^{2+}$-dependent. The same pattern of $\mathrm{Ni}^{2+}$-dependence dominance has been found in other plant species, two out of three Arabidopsis GLYI proteins are $\mathrm{Ni}^{2+}$-activated [11]. In addition, according to this study seven out of 10 soybean GLYI-like proteins and four out of five $M$. truncatula GLYI proteins are predicted to be $\mathrm{Ni}^{2+}$-activated (Additional file 1: Table S1). Moreover, previous reports have detected the presence of two GLYI domains in a single protein from Saccharomyces cerevisiae, Plasmodium falciparum and O. sativa [13, 66, 67]. Two domains could form two active sites in a single protein and both active sites were found to play roles in P. falciparum but one of the active sites was found to have no function in $O$. sativa [13, 67]. Here, VvGLYI-like1 and VvGLYI-like4 had two conserved glyoxalase domains (PF00903) (Fig. 1). They also possess four conserved metal binding residues in the $\mathrm{N}$ - terminal and three in the $\mathrm{C}$ - terminal (Additional file 5: Figure S1 and Additional file 9: Figure S4). That is the Cterminal domain of VvGLYIlike-1 and VvGLYI-like4 may have no function, as in rice. Similarly, GLYII enzymes also require divalent cations for their activity. GLYII proteins of plants, usually possess a binuclear metal binding center $[16,18,20,23]$. AtGLYII-5 is reported to have a predominant metal center of $\mathrm{Fe}(\mathrm{III}) \mathrm{Zn}(\mathrm{II})$ but does not appear to bind Mn specifically [18]. However, AtGLYII-2 has been shown to bind a mixture of $\mathrm{Zn}, \mathrm{Fe}$ or $\mathrm{Mn}$ and it also exhibits positive cooperativity in metal binding [21]. The gene dependence on metal ions still needs to be proved by experiments. We just predicted the metal dependence, providing some ideas for future research.

Previous characterisations of GLYIII activity in all species display a metal ion-independent nature $[5,6]$. However,
Hsp31 and human DJ-1 as glyoxalases reveal the existence of the essential catalytic residues, glutamic acid and cysteine, together with a histidine residue that assists catalysis $[4,5]$. However, the histidine positioning in DJ-1 and Hsp31 are different. In the present study, both domains in the N-terminal and C-terminal of VvGLYIII-like2 resembled Hsp31, AtDJ-1d and OsDJ-1C in that the cysteine and histidine were contiguous (Additional file 7: Figure S3). These proteins are reported to be important detoxification GLYIII enzymes in Arabidopsis, rice and E. coli [6, 7]. Thus we predict that VvGLYIII-like2 could have the same functions as these and might play important roles as a GLYIII in grape. In contrast, the N-terminal of VvGLYIIIlike-3 resembled that of Drosophila in which histidine is replaced by tyrosine but the histidine in the C-terminal of VvGLYIII-like3 was replaced by phenylalanine [68]. However, the N-terminal and C-terminal of VvGLYIII-like1 are in the same as human DJ-1protein (Additional file 7: Figure S3). Although the catalytic core is similar, catalytic activity was quite variable among the members of the GLYIII family and this suggests a variety of function for the members in this family.

\section{Different expression patterns of glyoxalase genes in response to stress}

The role of the glyoxalase system in resistance to multiple biotic and abiotic stresses has been well characterised in some studies. Recently, it has been reported that the glyoxalase pathway of rice not only increases tolerance to salinity, drought and extreme temperatures but also reduces damage from sheath blight fungus (Rhizoctonia solani) [69]. Moreover, some glyoxalase members of soybean such as GmGLYI-6/9/20 and GmGLYII-6/10 show special expression patterns in response to various pathogenic infection [9]. However, whether glyoxalase proteins participate in downy mildew response is unknown. In this study, we found that after downy mildew inoculation, most glyoxalase genes had two periods of high expression at 6-12 $\mathrm{h}$ and at 96-120 h. In particular, VvGLYII-like1 had a down-regulated expression pattern after downy mildew inoculation. After fungal infection, intercellular MG levels are significantly enhanced in susceptible genotypes, so high GLYI activity is needed [5]. Nevertheless, a rice GLYI gene was found to be down-regulated after infection with Xanthomonas oryzae pv. oryzae or with Pyricularia grisea [38]. Like PR1 and NPR1, VvGLYI-like1 was highly expressed $48 \mathrm{~h}$ after inoculation. Thus, VvGLYI-like1 may play an important role in the defence response to downy mildew. Whether the other glyoxalase-like genes are involved in defence responses still needs to be clarified. The infection process of $P$. viticola is complex and takes a long time, the expression levels of genes during this process are very variable. Further studies are required to 
determine if over expression of glyoxalase genes could offer direct protection to plants from pathogens.

Moreover, the high identity of glyoxalase genes between grape and Arabidopsis at the protein level indicates that glyoxalase genes may have similar conserved functions to their Arabidopsis orthologue genes (Additional file 10: Table S6). As with the grape glyoxalase-like genes, AtGLYI-2, AtGLYI-3, AtGLYI-6, AtGLYII-2 and AtGLYII-5 are highly expressed at all the stages of development [8]. The frequency of ESTs or cDNAs available in different databases has been considered as a useful tool for preliminary analysis of gene expression [70]. The search for ESTs was performed by comparing the grape glyoxalase cDNA sequences against EST sequences available at NCBI in BLASTN searches with the search parameters: maximum identity, > 95\%; length, $>300$ bp; and E value, $<10^{-10}$ [71]. The ESTs were identified for all of the GLY genes, indicating they are expressed. However, the frequency of ESTs was low, indicating that these are expressed at very low levels. The matched EST sequences were derived from various grape tissues or libraries such as root, leaf, berry, seed, bud, flower, pericarp, shoot, clusters and stem, indicating the differential expression of VvGLY-likes in grape tissues (Additional file 11: Table S7).

\section{Conclusions}

This study identifies four GLYI-like, two GLYII-like and three GLYIII-like glyoxalase proteins in grape. All these newly-identified members were further analysed for their homology, essential binding sites for catalytic activity, structural properties, expressions in different tissues and expressions under stress from downy mildew infection. This study lays a foundation for researching the roles of GLY genes of grape in response to downy mildew stress. Most glyoxalase genes were highly expressed at 6-12 h and 96-120 h post inoculation but VvGLYII-like1 expression was down-regulated. In particular, $V v G L Y I$-like1 was highly expressed $48 \mathrm{~h}$ after inoculation and, like PR1 and NPR1, might be involved in defence response to downy mildew. Despite many recent advances in functional studies of glyoxalase genes, the biological functions of most glyoxalase genes in physiological and developmental processes or plant defence still require elucidation. The bioinformatic analysis and expression patterns of the grape glyoxalase gene families conducted in our study provide an overview of the composition and expression of glyoxalase genes in grapevine that will facilitate selection of candidate genes for cloning and for further functional characterisation.

\section{Methods}

\section{Identification of glyoxalase genes in grape}

To identify the GLYI, GLYII and GLYIII genes in grape, the Hidden Markov Model (HMM) profiles of the glyoxalase domain (PF00903), metallo-beta-lactamase domain (PF00753) and DJ-1/PfpI (PF01965) were downloaded from the Pfam database (http://pfam.xfam.org/). They were then used as queries to search the Grape Genome Database (12X) (http://www.genoscope.cns.fr/ externe/GenomeBrowser/Vitis/) using HMMER3.1 with default E-values $(<1.0)$ [48]. All putative GLYI, GLYII and GLYIII genes were manually confirmed by searching the NCBI (https://www.ncbi.nlm.nih.gov/) and the Pfam program again with score value $\geq 100$ and e-value less than $10^{-30}$. To find the possibly active GLYI proteins, each of the identified VvGLYI-like protein sequences along with putative GLYI proteins in rice, Arabidopsis, soybean and $M$. truncatula was analysed using BLASTP at the score value $\geq 100$ and $E$ value $\leq 0$. Proteins with conserved binding sites, active sites, metal binding sites, GSH binging sites and dimer interfaces were assumed to be GLYI-like. The predicted proteins are named: prefix "Vv" for Vitis vinifera, followed by GLYI-like, GLYIIlike or GLYIII-like, and lastly the Arabic numbers (e.g. $1,2,3)$ based on their locations on the chromosome.

\section{Exon-intron and protein structure analysis}

The exon and intron of the glyoxalase-like genes were identified according to the Grape Genome Browser. Diagrams were drawn using the online tool GSDS 2.0 (http://gsds.cbi.pku.edu.cn) [72]. Coding sequences (CDs) and untranslated region (UTR) sequences of glyoxalase genes are provided in Additional files 16 . All the newly predicted grape glyoxalase-like proteins were analysed using Pfam (http://pfam.xfam.org) to reveal the position of domains.

\section{Multiple sequence alignment and phylogenetic analyses}

Two unrooted phylogenetic trees were constructed respectively with amino acid sequences of $\mathrm{N}$-terminal lactoylglutathione lyase domain (PF00903) in GLYI-like and full length of GLYII-like proteins from grape Arabidopsis, rice, soybean, M. truncatula using MEGA 5.1 with the Maximum Likelihood method and 1000 bootstrap replicates $[73,74]$. In addition, $\mathrm{N}$-terminal and C-terminal DJ-1/PfpI (PF01965) domain from grape Arabidopsis, rice, $M$. truncatula along with DJ-1/PfpI domain from human, mouse, Drosophila, C. elegans and Hsp31 from E. coli were used to construct another phylogenetic tree using the same method and the sequences are shown in Additional files 13, 14 and 15 respectively. Multiple sequence alignment was carried out using ClustalW [75] and visualized with Jalview [76].

\section{Expression of grape glyoxalase genes in different tissues and under downy mildew stress}

'Thompson Seedless' (Vitis vinifera L.) was grown in the vineyard of Northwest A\&F University, Yangling, Shaanxi, 
China and grapevines were managed according to local standards. Samples of roots, stems, leaves, flowers, tendrils and ovules were collected. Ovules were collected 20 DAF (small globular embryo), 30 DAF (globular embryo) and 40 DAF (aborted embryo). For the downy mildew treatment, downy mildew (Plasmopara viticola) infected leaves were collected from the vineyard. Sporangia were isolated using vacuum aspiration [77]. Detached healthy leaves of 'Thomson seedless' were obtained from the same position on the shoot (fifth to sixth unfolded leaf). These were surface sterilized with $70 \%$ (vol/vol) ethanol and then rinsed in deionised water. The abaxial leaf surfaces were inoculated with $100 \mu$ ldroplets of an aqueous suspension of sporangia $\left(2 \times 10^{6}\right.$ sporangia $\left.\mathrm{ml}^{-1}\right)$. The control plants were neither inoculated nor elicitor-treated. The petioles were then covered with a piece of wet cotton, incubated in the dark at $25^{\circ} \mathrm{C}$ and $>95 \%$ relative humidity (RH) [78]. Inoculated leaves were then collected after $0,6,12,24,48$, 72, 96 and $120 \mathrm{~h}$, these were immediately immersed in liquid nitrogen and stored at $-80^{\circ} \mathrm{C}$ pending analysis. All the plant materials were replicated three times.

\section{RNA isolation, qRT-PCR and statistical analysis}

The EZNA Plant RNA Kit (Omega, Guangzhou, China) was used to extract the total RNA following the manufacturer's instructions. For qRT-PCR (Quantitative Real-time PCR) analysis, the first-strand cDNA was synthesised using PrimeScript RTase (Takara, Dalian, China) according to the manufacturer's instructions. Primers were designed by Primer Premier 5.0 (Premier, Canada) and these are listed in Additional file 12: Table S8. The qRT-PCR was carried out using SYBR green (Takara, Dalian, China) with the IQ5 real time PCR system (Bio-Rad, Hercules, CA, USA). Grape Actin 7 (Accession no. XM_002282480) was used as an internal control. VvMADS9 (Accession no. NM_001280946), VvPR1 (Accession no. XM 002273752) and VvNPR1 (Accession no. XM_002281439) were used as a positive control. Three biological repeats were used and each reaction was carried out three times. The normalised expression method $\left(2^{-\triangle \Delta C(t)}\right.$ method) was used to analyse the relative expression levels with the highest level of expression set as 1. Statistical analyses were conducted using SPSS 18.0 Software (Chicago, IL) and Excel. Statistically significant analyses were based on Duncan's biological repeats and each reaction is reported as a mean with standard deviations (SDs) presented as error bars.

\section{Additional files}

Additional file 1: Table S1. Essential amino acids and metal ion dependency analysis of all putative GLYI proteins from Vitis vinifera, Arabidopsis, Oryza sativa, Glycine max and Medicago truncatula. (DOCX 15 kb)
Additional file 2: Table S2. Conserved binding sites analysis of genes previously reported as GLYI proteins of Arabidopsis, rice, soybean and Medicago truncatula that are not likely to be GLYs. (DOC $94 \mathrm{~kb}$ )

Additional file 3: Table S3. Conserved binding sites analysis of all putative GLYII proteins from Vitis vinifera, Arabidopsis, Oryza sativa, Glycine max and Medicago truncatula. (DOCX $14 \mathrm{~kb}$ )

Additional file 4: Table S4. Conserved binding sites analysis of genes previously reported as GLYII proteins of Arabidopsis, rice, soybean and Medicago truncatula that are not likely to be GLYs. (DOCX $16 \mathrm{~kb}$ )

Additional file 5: Figure S1. Multiple Sequence alignments of GLYI domains. N-terminal GLYI domains of proteins listed in Additional file 1: Table S1, along with a Gly I from human (Homo sapiens, Accession No: AB209801), were aligned using ClustalW and edited using the Jalview program. Four conserved residues (H/E/H/E) for metal binding are shown with black boxes and specific regions for $\mathrm{Zn}^{2+}$-dependence are in pink boxes. (DOCX $759 \mathrm{~kb}$ )

Additional file 6: Figure S2. Sequence alignments of full length of GLYIl proteins. Multiple sequence alignments were conducted with full length protein sequences listed in Additional file 3: Table S3 along with Glyll of human (Homo sapiens, Accession No: NP_005317) and a known GLYIl protein from Brassica juncea (B. juncea, Accession No: AY185202). The conserved active motif $\mathrm{G} / \mathrm{CHT}$ is indicated in black boxes. The conserved metal binding sites are marked with "\#" and the GSH binding sites are marked with red stars. (DOCX $781 \mathrm{~kb})$

Additional file 7: Figure S3. Sequence alignment of the N-terminal and C-terminal DJ-1/Pfpl domains of GLYIII-like proteins. Both the N-terminal and C-terminal DJ-1/Pfpl domains of VVGLYIII-like proteins were aligned with other well characterized DJ-1/Pfpl superfamily proteins from human, mouse, Drosophila, Caenorhabditis elegans, Escherichia coli and orthologs from Arabidopsis, rice, soybean and Medicago truncatula. Three residues relate to the activity are marked with black boxes. Proteins without the cysteine in the $\mathrm{N}$-terminal or $\mathrm{C}$-terminal are marked with red boxes respectively. (DOCX $1457 \mathrm{~kb}$ )

Additional file 8: Table S5. The number of previously reported glyoxalase genes and glyoxalase genes with the critical conserved binding sites in grape, rice, Arabidopsis, soybean and Medicago truncatula. (DOCX $17 \mathrm{~kb}$ )

Additional file 9: Figure S4. Multiple sequence alignment of C-terminal GLYI domain of VVGLYI-like1 and VVGLYI-like4. C-terminal GLYI domains of $V_{V G L Y}$-like1 and VVGLYI-like4 were aligned with the C-terminal GLYI domain of OsGLYI-11 and the N-terminal GLYI domain of AtGLYI-2 using ClustalW and then edited by the Jalview program. All four conserved metal binding sites are shown in black boxes. (DOCX $247 \mathrm{~kb}$ )

Additional file 10: Table S6. Percent Identity Matrix of GLY-like families bweteen grape and Arabidopsis. (DOCX $23 \mathrm{~kb}$ )

Additional file 11: Table S7. Expression analysis of VVGLY-like genes in the ESTs database. (DOCX $14 \mathrm{~kb}$ )

Additional file 12: Table S8. Primers used in expression analysis of glyoxalase-like gene families in grape. (DOCX $18 \mathrm{~kb}$ )

Additional file 13: Amino acid sequences of lactoylglutathione lyase domains and full length protein sequences of GLYIs used for phylogenetic analysis and multiple sequence alignment. (DOCX $22 \mathrm{~kb}$ )

Additional file 14: Amino acid sequences of full length putative GLYlls used for phylogenetic analysis and multiple sequence alignment. (DOCX $17 \mathrm{~kb}$ )

Additional file 15: Amino acid sequences of DJ-1/Pfpl domains and full length sequences of DJ-1 proteins used for phylogenetic analysis. (DOCX $25 \mathrm{~kb}$ )

Additional file 16: Other sequences and their accession numbers used in this paper. (DOCX $58 \mathrm{~kb})$

\section{Abbreviations}

CDS: Coding sequence; DAF: Day after flowering; GLYI: Glyoxalase I:

GLYII: Glyoxalase II; GLYIII: Glyoxalase III; GSDS: Gene Structure Display Server; GSH: reduced glutathione; HMM: Hidden Markov Model; HTA: Hemithioacetal; Mb: Mega base; MG: Methylglyoxal; ML: Maximum Likelihood; qRTPCR: Quantitative Real-time PCR; RH: Relative humidity; SLG: S-D-lactoylglutathione; UTR: Untranslated region 


\section{Acknowledgements}

We thank reviewers for their helpful comments and the editors for their careful work on the manuscript.

\section{Funding}

This research was supported by the National Natural Science Foundation of China (Grant No. 31471844) and National Key Research and Development Program of China (2008YFD1000300). The funding bodies had no role in the design of this study and collection, analysis, and interpretation of data and in writing the manuscript.

\section{Availability of data and materials}

All sequences analysed during this study are included in this published article [and its supplementary information files]. The raw data of qRT-PCR in current study can be available from the corresponding author on reasonable request.

\section{Authors' contributions}

$Y X$ and $Y J W$ conceived and designed the experiments. TM L, XC, YT W, XY performed the experiments. ZQ $L$ and $R Q L$ prepared the plant materials and treated the samples. GT $L$ revised the paper. TM $L$ analysed the data and wrote the manuscript. All authors read and approved the final manuscript.

\section{Ethics approval and consent to participate}

Not applicable.

\section{Consent for publication}

Not applicable.

\section{Competing interests}

The authors declare they have no competing interests.

\section{Publisher's Note}

Springer Nature remains neutral with regard to jurisdictional claims in published maps and institutional affiliations.

\section{Author details}

${ }^{1}$ College of Horticulture, Northwest A\&F University, Yangling, Shaanxi, People's Republic of China. ${ }^{2}$ State Key Laboratory of Crop Stress Biology for Arid Areas, Northwest A\&F University, Yangling, Shaanxi, People's Republic of China. ${ }^{3}$ Key Laboratory of Horticultural Plant Biology and Germplasm Innovation in Northwest China, Ministry of Agriculture, Yangling, Shaanxi, People's Republic of China.

Received: 22 March 2018 Accepted: 24 April 2019

Published online: 09 May 2019

\section{References}

1. Racker E. The mechanism of action of glyoxalase. J Biol Chem. 1951;190(2): 685-96.

2. Thornalley PJ. The glyoxalase system: new developments towards functional characterization of a metabolic pathway fundamental to biological life. Biochem J. 1990;269(1):1-11.

3. Zhao QQ, Su Y, Wang ZK, Chen CP, Wu TSY, Huang Y. Identification of glutathione (GSH)-independent glyoxalase III from Schizosaccharomyces pombe. BMC Evol Biol. 2014;14(1):86.

4. Subedi KP, Choi D, Kim I, Min B, Park C. Hsp31 of Escherichia coli k-12 is glyoxalase III. Mol Microbiol. 2011;81(4):926-36.

5. Lee JY, Song J, Kwon K, Jang S, Kim C, Baek K, Kim J, Park C. Human DJ-1 and its homologs are novel glyoxalases. Hum Mol Genet. 2012;21 (14):3215-25.

6. Kwon K, Choi D, Hyun J, Jung H, Baek K, Park C. Novel glyoxalases from Arabidopsis thaliana. FEBS J. 2013;280(14):3328-39.

7. Ghosh A, Kushwaha HR, Hasan MR, Pareek A, Sopory SK, Singla-Pareek SL. Presence of unique Gyoxalase III proteins in plants indicates the existence of shorter route for methylglyoxal detoxification. Sci Rep 2016;6(18358):18358.

8. Mustafiz A, Singh AK, Pareek A, Sopory SK, Singla-Pareek SL. Genomewide analysis of rice and Arabidopsis identifies two glyoxalase genes that are highly expressed in abiotic stresses. Funct Integr Genomics. 2011;11(2):293-305.

9. Ghosh A, Islam T. Genome-wide analysis and expression profiling of glyoxalase gene families in soybean (Glycine max) indicate their development and abiotic stress specific response. BMC Plant Biol. 2016;16(1):87.
10. Ghosh A. Genome-wide identification of glyoxalase genes in Medicago truncatula and their expression profiling in response to various developmental and environmental stimuli. Front Plant Sci. 2017;8:836.

11. Jain M, Batth R, Kumari S, Mustafiz A. Arabidopsis thaliana contains both $\mathrm{Ni}^{2+}$ and $\mathrm{Zn}^{2+}$ dependent glyoxalase I enzymes and ectopic expression of the latter contributes more towards abiotic stress tolerance in E. coli. PLoS One. 2016;11(7):e0159348.

12. Kaur C, Vishnoi A, Ariyadasa TU, Bhattacharya A, Singla-Pareek SL, Sopory SK. Episodes of horizontal gene-transfer and gene-fusion led to co-existence of different metal-ion specific glyoxalase I. Sci Rep. 2013;3(11):3076.

13. Mustafiz A, Ghosh A, Tripathi AK, Kaur C, Ganguly AK, Bhavesh NS, Tripathi JK, Pareek A, Sopory SK, Singla-Pareek SL. A unique $\mathrm{Ni}^{2+}$-dependent and methylglyoxal-inducible rice glyoxalase I possesses a single active site and functions in abiotic stress response. Plant J. 2014;78:951-63.

14. Kaur C, Tripathi AK, Nutan KK, Sharma S, Ghosh A, Tripathi JK, Pareek A, Singla-Pareek SL, Sopory SK. A nuclear-localized rice glyoxalase I enzyme, OsGLYI-8, functions in the detoxification of methylglyoxal in the nucleus. Plant J. 2017;89:565-76.

15. Maiti MK, Krishnasamy S, Owen HA, Makaroff CA. Molecular cloning of glyoxalase II from a higher plant: comparison of mitochondrial and cytoplasmic isozymes. Plant Mol Biol. 1997;35:471-81.

16. Limphong P, McKinney R, Adams N, Makaroff C, Bennett B, Crowder M. The metal ion requirements of Arabidopsis thaliana GIx2-2 for catalytic activity. J Biol Inorg Chem. 2010;15(2):249-58.

17. Crowder MW, Maiti MK, Banovic L, Makaroff CA. Glyoxalase II from A. thaliana requires Zn(II) for catalytic activity. FEBS Lett. 1997;418(3):351-4.

18. Marasinghe GPK, Sander IM, Bennett B, Periyannan G, Yang KW, Makaroff CA, Crowder MW. Structural studies on a mitochondrial glyoxalase II. J Biol Chem. 2005;280:40668-75.

19. Singla-Pareek SL, Reddy MK, Sopory SK. Genetic engineering of the glyoxalase pathway in tobacco leads to enhanced salinity tolerance. Proc Natl Acad Sci U S A. 2003;100(25):14672-7.

20. Ghosh A, Pareek A, Sopory SK, Singla-Pareek SL. A glutathione responsive rice glyoxalase II, OsGLYII-2, functions in salinity adaptation by maintaining better photosynthesis efficiency and anti-oxidant pool. Plant J. 2014;80:93-105.

21. Wenzel NF, Carenbauer AL, Pfiester MP, Schilling O, Meyer-Klaucke W, Makaroff CA, Crowder MW. The binding of iron and zinc to glyoxalase II occurs exclusively as di-metal centers and is unique within the metallo- $\beta$ lactamase family. J Biol Inorg Chem. 2004;9:429-38.

22. Melino S, Capo C, Dragani B, Aceto A, Petruzzelli R. A zinc-binding motif conserved in glyoxalase II, $\beta$-lactamase and arylsulfatases. Trends Biochem Sci. 1998;23(10):381.

23. Schilling O, Wenzel N, Naylor M, Vogel A, Crowder M, makaroff C, MeyerKlaucke W. Flexible metal binding of the metallo-beta-lactamase domain: glyoxalase II incorporates iron, manganese, and zinc in vivo. Biochemistry. 2003;42(40):11777-86.

24. Misra K, Banerjee AB, Ray S, Ray M. Glyoxalase III from Escherichia coli: A single novel enzyme for the conversion of methylglyoxal into D-lactate without reduced glutathione. Biochem J. 1995;305 Pt 3, 999-1003.

25. Smits MM, Johnson MA. Methylglyoxal: enzyme distributions relative to its presence in Douglas-fir needles and absence in Douglas-fir needle callus. Arch Biochem Biophys. 1981;208(2):431-9.

26. Talesa V, Rosi G, Contenti S, Mangiabene C, Lupattelli M, Norton SJ, Giovannini E, Principato GB. Presence of glyoxalase II in mitochondria from spinach leaves: comparison with the enzyme from cytosol. Biochem Int. 1990;22:1115-20.

27. Norton SJ, Talesa V, Yuan WJ, Principato GB. Glyoxalase I and glyoxalase II from Aloe vera: purification, characterization and comparison with animal glyoxalases. Biochem Int. 1990;22:411-8.

28. Deswal R, Chakaravarty TN, Sopory SK. The glyoxalase system in higher plants: regulation in growth and differentiation. Biochem Soc Trans. 1993;21 (2):527-30.

29. Paulus $C$, Kollner B, Jacobsen HJ. Physiological and biochemical characterization of glyoxalase I, a general marker for cell proliferation, from a soybean cell suspension. Planta. 1993;189:561-6.

30. Chakravarty TN, Sopory SK. Blue light stimulation of cell proliferation and glyoxalase I activity in callus cultures of Amaranthus paniculatus. Plant Sci. 1998;132:63-9.

31. Kaur C, Singla-Pareek SL, Sopory SK. Glyoxalase and methylglyoxal as biomarkers for plant stress tolerance. Crit Rev Plant Sci. 2014;33(6):429-56.

32. Veena RVS, Sopory SK. Glyoxalase I from Brassica juncea: molecular cloning, regulation and its over-expression confer tolerance in transgenic tobacco under stress. Plant J. 1999;17:385-95. 
33. Lin F, Xu J, Shi J, Li H, Li B. Molecular cloning and characterization of a novel glyoxalase I gene TaGly / in wheat (Triticum aestivum L.). Mol Biol Rep. 2010;37(2):729-35.

34. Chakrabarty D, Trivedi PK, Misra P, Tiwari M, Shri M, Shukla D, Kumar S, Rai A, Pandey A, Nigam D, Tripathi RD, Tuli R. Comparative transcriptome analysis of arsenate and arsenite stresses in rice seedlings. Chemosphere. 2009;74(5):688-702

35. Saxena M, Bisht R, Roy SD, Sopory SK, Bhalla-Sarin N. Cloning and characterization of a mitochondrial Glyoxalase // from Brassica juncea that is upregulated by $\mathrm{NaCl}$, Zn, and ABA. Biochem Biophys Res Commun. 2005;336(3):813-9.

36. Yadav SK, Singla-Pareek SL, Kumar M, Pareek A, Saxena M, Sarin NB, Sopory SK. Characterization and functional validation of Glyoxalase // from rice. Protein Expr Purif. 2007;51:126-32

37. Sankaranarayanan S, Jamshed M, Kumar A, et al. Glyoxalase Goes green: the expanding roles of glyoxalase in plants. Int J Mol Sci. 2017;18(4):898.

38. Zhou B, Peng KM, Chu ZH, Wang SP, Zhang QF. The defense-responsive genes showing enhanced and repressed expression after pathogen infection in rice (Oryza sativa L.). Sci China C Life Sci. 2002;45(5):449-67.

39. Chen ZY, Brown RL, Damann KE, Cleveland TE. Identification of a maize kernel stress-related protein and its effect on aflatoxin accumulation. Phytopathology. 2004;94(9):938-45.

40. Sangha JS, Chen YH, Kaur J, Khan W, Abduljaleel Z, Alanazi MS, Mills A, Adalla BC, Bennett J, Prithiviraj B, Jahn GC, Leung H. Proteome analysis of rice (Oryza sativa L.) mutants reveals differentially induced proteins during brown planthopper (Nilaparvata lugens) infestation. Int J Mol Sci. 2013;14(2):3921-45.

41. Jaillon O, Aury JM, Noel B, Policriti A, Clepet C, Casagrande A, Choisne N, Aubourg S, Vitulo N, Jubin C, Vezzi A, et al. The grapevine genome sequence suggests ancestral hexaploidization in major angiosperm phyla. Nature. 2007;449(7161):463-5.

42. Pearson RC, Goheen AC. Compendium of grape diseases. Mycologia. 1988;81(1):176.

43. Merdinoglu D, Wiedemann-Merdinoglu S, Coste P, Dumas V, Haetty S, Butterlin G, Greif C. Genetic analysis of downy mildew resistance derived from Muscadinia rotundifolia. Acta Hort. 2003;603(603):451-6.

44. Henanff GL, Heitz T, Mestre P, Mutterer J, Walter B, Chong J. Characterization of Vitis vinifera NPR1 homologs involved in the regulation of pathogenesis-related gene expression. BMC Plant Biol. 2009;9:54-67.

45. Kortekamp A. Expression analysis of defence-related genes in grapevine leaves after inoculation with a host and a non-host pathogen. Plant Physiol Biochem. 2006;44:58-67.

46. Su H, Jiao YT, Wang FF, Liu YE, Niu WL, Liu GT, Xu Y. Overexpression of VpPR10.1 by an efficient transformation method enhances downy mildew resistance in V. vinifera. Plant Cell Rep. 2018:37:819-32.

47. Ma H, Xiang GQ, Li ZQ, Wang YT, Dou MR, Su L, Yin X, Liu RQ, Wang YJ, Xu $Y$. Grapevine VpPR10.1 functions in resistance to Plasmopara viticola through triggering a cell death-like defense response by interacting with VpVDAC3. Plant Biotechnol J. 2018;16:1488-501.

48. Eddy SR. Profile hidden Markov models. Bioinformatics. 1998;14(9):755-63.

49. Limphong P, Crowder MW, Bennett B, Makaroff CA. Arabidopsis thaliana GLX2-1 contains a dinuclear metal binding site, but is not a glyoxalase 2 . Biochem J. 2009;417(1):323-30.

50. Ridderstrom M, Saccucci F, Hellman U, Bergman T, Principato G, Mannervik B. Molecular cloning, heterologous expression, and characterization of human glyoxalase II. J Biol Chem. 1996;271:319-23.

51. Limphong P, Nimako G, Thomas PW, Fast W, Makaroff CA, Crowder MW. Arabidopsis thaliana mitochondrial glyoxalase 2-1 exhibits $\beta$-lactamase activity. Biochemistry. 2009;48(36):8491.

52. Shin SH, Sun SC. Lengths of coding and noncoding regions of a gene correlate with gene essentiality and rates of evolution. Genes Genom. 2015;37(4):365-74.

53. Cameron AD, Ridderström M, Olin B, Mannervik B. Crystal structure of human glyoxalase II and its complex with a glutathione thiolester substrate analogue. Structure. 1999;7(9):1067-78.

54. Kaur C, Sharma S, Hasan MR, Pareek A, Singla-Pareek SL, Sopory SK. Characteristic variations and similarities in biochemical, molecular, and functional properties of glyoxalases across prokaryotes and eukaryotes. Int J Mol Sci. 2017;18(4):250.

55. Ridderstrom M, Cameron AD, Jones TA, Mannervik B. Involvement of an active-site $\mathrm{Zn}^{2+}$ ligand in the catalytic mechanism of human glyoxalase I. J Biol Chem. 1998;273(34):21623-8.

56. Islam T, Ghosh A. Genome-wide dissection and expression profiling of unique glyoxalase III genes in soybean reveal the differential pattern of transcriptional regulation. Sci Rep-UK. 2018;8(1):4848.
57. Xu XM, Lin H, Maple J, Bjorkblom B, Alves G, Larsen JP, Moller SG. The Arabidopsis DJ-1a protein confers stress protection through cytosolic SOD activation. J Cell Sci. 2010;123:1644-51.

58. Sreekantan $\mathrm{L}$, Torregrosa $\mathrm{L}$, Fernandez L, Thomas MR. VVMADS9, a class B MADS-box gene involved in grapevine flowering, shows different expression patterns in mutants with abnormal petal and stamen structures. Funct Plant Biol. 2006;33(9):877-86.

59. Iskandar HM, Simpson RS, Casu RE, Bonnett GD, Maclean DJ, Manners JM. Comparison of reference genes for quantitative real-time polymerase chain reaction analysis of gene expression in sugarcane. Plant Mol Biol Rep. 2004;22(4):325-37.

60. Jaillon O, Aury JM, Noel B, Policriti A, Clepet C, Casagrande A, Choisne N, Aubourg S, Vitulo N, Jubin C, Vezzi A, et al. The grapevine genome sequence suggests ancestral hexaploidization in major 48 angiosperm phyla. Nature. 2007;449(7161):463-5.

61. Yu J, Hu SN, Wang J, Wong GK, Li S, Liu B, Deng YJ, Dai L, Zhou Y, Zhang $X Q$, et al. A draft sequence of the rice genome (Oryza sativa L. ssp. indica). Science. 2002;296(5565):79-92.

62. Bennett MD, Leitch IJ, Price HJ, Johnston JS. Comparisons with Caenorhabditis ( 100 Mb) and Drosophila ( 175 Mb) using flow cytometry show genome size in Arabidopsis to be $\sim 157 \mathrm{Mb}$ and thus $\sim 25 \%$ larger than the Arabidopsis genome initiative estimate of 125 Mb. Ann Bot. 2003;91(5):547-57.

63. Schmutz J, Cannon SB, Schlueter J, Ma J, Mitros T, Nelson W, Hyten D, Song QJ, Thelen JJ, Cheng JL, et al. Genome sequence of the palaeopolyploid soybean. Nature. 2010;463(7278):178-83.

64. Young ND, Debellé F, Oldroyd GE, Geurts R, Cannon SB, Udvardi MK, Benedito VA, Mayer KF, Gouzy J, Schoof H, et al. The Medicago genome provides insight into the evolution of rhizobial symbioses. Nature. 2011;480(7378):520-4.

65. Suttisansanee U, Lau K, Lagishetty S, Rao KN, Swaminathan S, Sauder JM, Burley SK, Honek JF. Structural variation in bacterial glyoxalase I enzymes: investigation of the metalloenzyme glyoxalase I from Clostridium acetobutylicum. J Biol Chem. 2011;286(44):38367-74.

66. Frickel EM, Jemth P, Widersten M, Mannervik B. Yeast glyoxalase I is a monomeric enzyme with two active sites. J Biol Chem. 2001;276(3):1845-9.

67. Deponte M, Sturm N, Mittler S, Harner M, Mack H, Becker K. Allosteric coupling of two different functional active sites in monomeric Plasmodium falciparum glyoxalase I. J Biol Chem. 2007;282(39):28419-30.

68. Meulener M, Whitworth AJ, Armstrong-Gold CE, Rizzu P, Heutink P, Wes PD, Pallanck LJ, Bonini NM. Drosophila DJ-1 mutants are selectively sensitive to environmental toxins associated with Parkinson's disease. Curr Biol. 2005; 15(17):1572-7.

69. Gupta BK, Sahoo KK, Ghosh A, Tripathi AK, Anwar K, Das P, Singh AK, Pareek A, Soopory SK, Singla-Pareek SL. Manipulation of glyoxalase pathway confers tolerance to multiple stresses in rice. Plant Cell Environ. 2017;41(5):1186-200.

70. Adams MD, Kerlavage AR, Fleischmann RD, Fuldner RA, Bult CJ, Lee NH, Kirkness EF, Weinstock KG, Gocayne JD, White O. Initial assessment of human gene diversity and expression patterns based upon 83 million nucleotides of cDNA sequence. Nature. 1995;377:3-17.

71. Jiang HY, Wu QQ, Jin J, Sheng L, Yan HW, Cheng BJ, Zhu SW. Genome-wide identification and expression profiling of ankyrin-repeat gene family in maize. Dev Genes Evol. 2013;223(5):303-18.

72. Hu B, Jin J, Guo AY, Zhang H, Luo JC, Gao G. GSDS 2.0: an upgraded gene feature visualization server. Bioinformatics. 2015;31(8):1296.

73. Cao J, Han X, Zhang TC, Yang YP, Huang JL, Hu XY. Genome-wide and molecular evolution analysis of the subtilas gene family in Vitis vinifera. BMC Genomics. 2014;15:1116.

74. Tamura K, Peterson D, Peterson N, Stecher G, Nei M, Kumar S. MEGA5: molecular evolutionary genetics analysis using maximum likelihood, evolutionary distance, and maximum parsimony methods. Mol Biol Evol. 2011;28(10):2731-9.

75. Larkin MA, Blackshields G, Brown NP, Chenna R, Mcgettigan PA, Mcwilliam H, Valentin F, Wallace IM, Wilm A, Lopez R, Thompson JD, Gibson TJ, Higgins DG. Clustal W and Clustal X version 2.0. Bioinformatics. 2007;23(21):2947-8.

76. Waterhouse AM, Procter JB, Martin DM, Clamp M, Barton GJ. Jalview version 2-a multiple sequence alignment editor and analysis workbench. Bioinformatics. 2009;25(9):1189-91.

77. Wong FP, Wilcox WF. Distribution of baseline sensitivities to azoxystrobin among isolates of plasmopara viticola. Plant Dis. 2000;84(3):275-81.

78. Unger S, Büche C, Boso S, Kassemeyer HH. The course of colonization of two different Vitis genotypes by Plasmopara viticola indicates compatible and incompatible host-pathogen interactions. Phytopathology. 2007;97(7):780-6. 